\title{
BONE SCANNING OF THE FOOT FOR UNEXPLAINED PAIN
}

\author{
H. D. MAURICE, J. H. NEWMAN, I. WATT \\ From Bristol Royal Infirmary, Bristol
}

\begin{abstract}
This paper reports the results of bone scans on 78 painful feet. Scanning helped in the diagnosis of persistent foot pain following injury and it enabled stress fractures, fractures of the sesamoids and subtalar arthritis to be diagnosed earlier. It reliably excluded bone infection and was useful as a screening test when radiographs were normal.
\end{abstract}

Many publications have stressed the value of isotope scans in the diagnosis of bone and joint pathology (Hughes 1980; Galasko and Weber 1984). There is, however, very little guidance as to the value of scintigraphy in the diagnosis of the painful foot. Goldman, Pavlov and Schneider (1982) commented on the usefulness of scintigraphy in the diagnosis of subtalar coalitions; they also reviewed 100 patients who were investigated for painful feet and found that many of them had normal scans. Others (Tannenbaum, Esdaile and Rosenthall 1980; Sewell et al. 1980; Kozin et al. $1976 \mathrm{a}, \mathrm{b})$ have presented small series, commenting on the use of scanning in plantar fasciitis, regional migratory osteoporosis and reflex sympathetic dystrophy.

This paper attempts to establish the value of isotope bone scanning in the diagnosis of unexplained foot pain.

\section{MATERIAL AND METHODS}

All patients who had a bone scan of the feet performed at the Bristol Royal Infirmary between January 1982 and June 1985 and whose clinical case notes and plain radiographs were available for examination, are included in this study. The scan was performed with a gamma-camera after injection of technetium-99m hydroxymethylene diphosphonate (HDP) in a dose of $600 \mathrm{MBq}$. Blood pool images were obtained from the first 300000 counts after injection and a delayed image two to three hours later.

There were 78 patients; 41 had a foot scan requested because of pain following trauma; the remaining $37 \mathrm{had}$ scans requested for non-traumatic conditions. For each

H. D. Maurice, FRCS, Senior Orthopaedic Registrar King's College Hospital, Denmark Hill, London SE5 9RS, England.

J. H. Newman, FRCS, Consultant Orthopaedic Surgeon

I. Watt, MRCP, FRCR, Consultant Radiologist

Bristol Royal Infirmary, Bristol BS2 8HW, England.

Requests for reprints should be sent to Mr H. D. Maurice.

(C) 1987 British Editorial Society of Bone and Joint Surgery $0301-620 \mathrm{X} / 87 / 3093 \$ 2.00$ patient, the notes, radiographs and foot scans were reviewed, the diagnosis was recorded and the part played by the bone scan in reaching the diagnosis was assessed.

\section{RESULTS}

\section{Trauma}

Persistent pain. Twenty-two patients were investigated for persistent pain following relatively minor injury. Two distinct patterns of isotope scan were obtained. The first was a generalised increase in activity, seen in patients who had been treated by plaster immobilisation for a fracture in the foot and who, on removal of the plaster, had complained of persistent pain. Their clinical features varied from relatively normal to a full-blown reflex sympathetic dystrophy, and plain radiographs showing juxta-articular osteoporosis of varying severity. Scans of those with sympathetic dystrophy (two patients) showed a confluent zone of increased activity extending over a large area (Figs 1, 2 and 3). Those with less severe symptoms had more patchy uptake representing the more usual disuse osteoporosis; pain settled more quickly in this group (Fig. 4).

The second pattern seen was a localised increased activity in the absence of any abnormality on the plain radiograph. Twelve patients who were sportsmen with ankle injuries had plain radiographs which failed to show any bony abnormalities. On returning to full activity they complained of persistent pain. Foot scans revealed localised activity thought to indicate major capsular or ligamentous damage (Figs 5 and 6).

Sesamoid fractures. Six patients complained of pain in the region of the great toe after injury; plain radiographs showed a divided sesamoid. In four of these the scan showed increased activity over the affected sesamoid; this was thought to indicate a fresh fracture, a diagnosis which was confirmed in two patients who were surgically explored (Figs 7 and 8). In two the scan was normal, suggesting a congenital bipartite sesamoid; one of these had a similar radiographic anomaly in the other foot. 


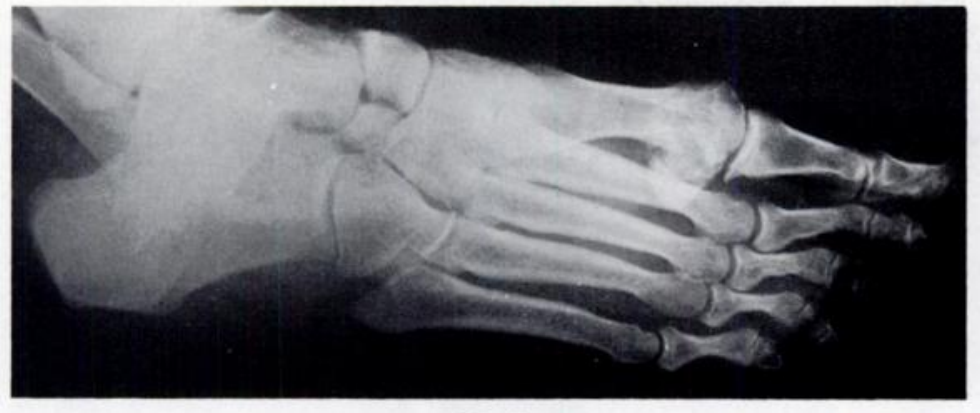

Fig. 1

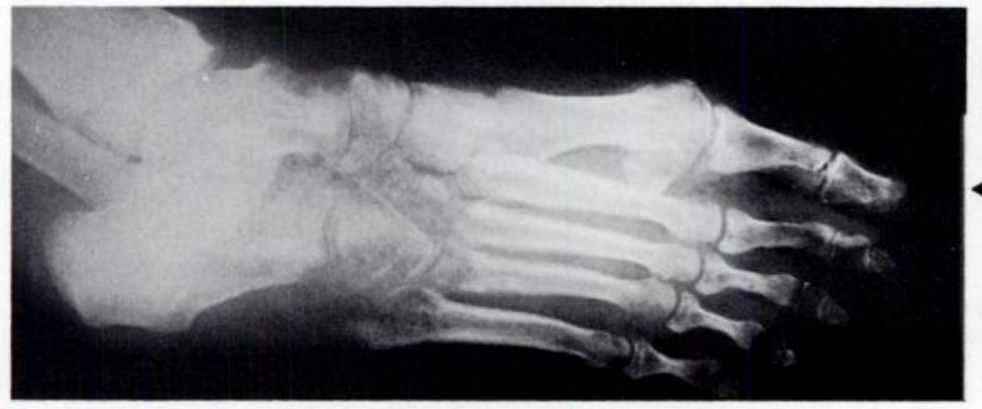

Fig. 2

Case 1. Figure 1 - A 53-year-old woman with a fractured navicular treated in plaster for six weeks. Figure 2 - Out of plaster her foot was painful and swollen with widespread juxta-articular osteoporosis. Figure 3 - A confluent zone of increased activity on the bone scan indicating reflex sympathetic dystrophy.

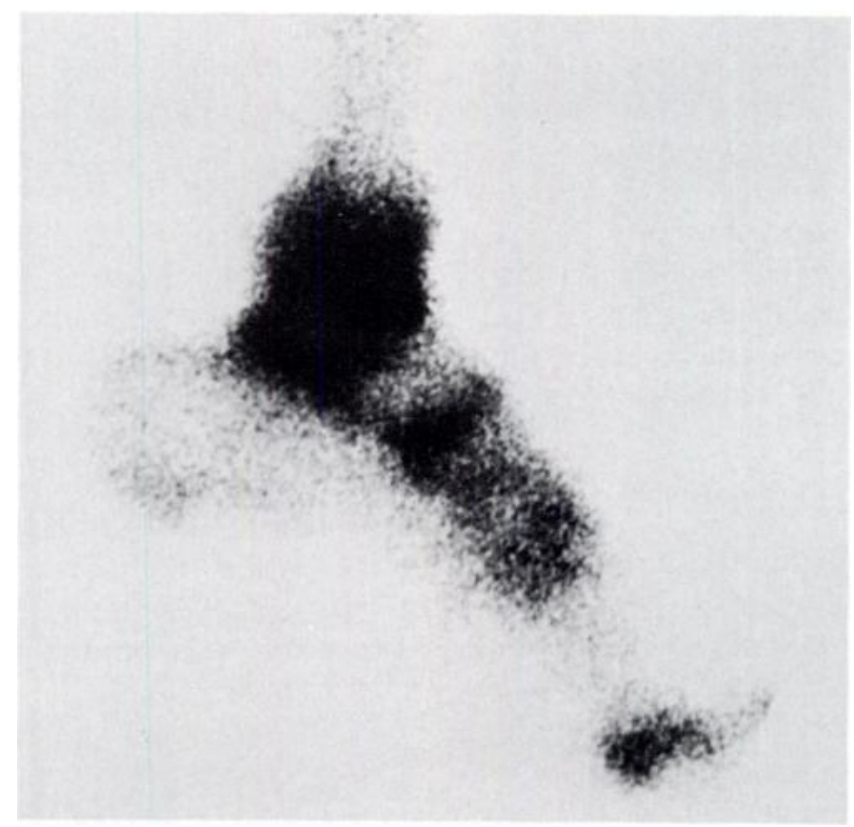

Fig. 4

Case 2. Patchy uptake on the foot scan in a 40-year-old man following plaster immobilisation for a tibial fracture - disuse osteoporosis.

Stress fractures. Four patients were scanned to search for stress fractures before radiographic changes. Two had positive scans and subsequent radiographs confirmed fractures two and three weeks later.

Subtalar arthritis. Five patients complained of persistent pain following healing of a hindfoot fracture. Plain radiographs, whilst confirming that bony union had occurred, did not reveal evidence of subtalar arthritis. Four had positive bone scans with maximal activity over the subtalar joint rather than over the healed fracture, indicating that in fact they had subtalar arthritis (Figs 9, 10 and 11). The fifth patient had a normal scan and his symptoms settled.

Miscellaneous. Scans were requested in two patients to confirm that, after fractures, the talus was not avascular: these, however, were requested too long after the injury to be of use. Two patients had scans to confirm union of a subtalar arthrodesis, undertaken because of osteoarthri- 


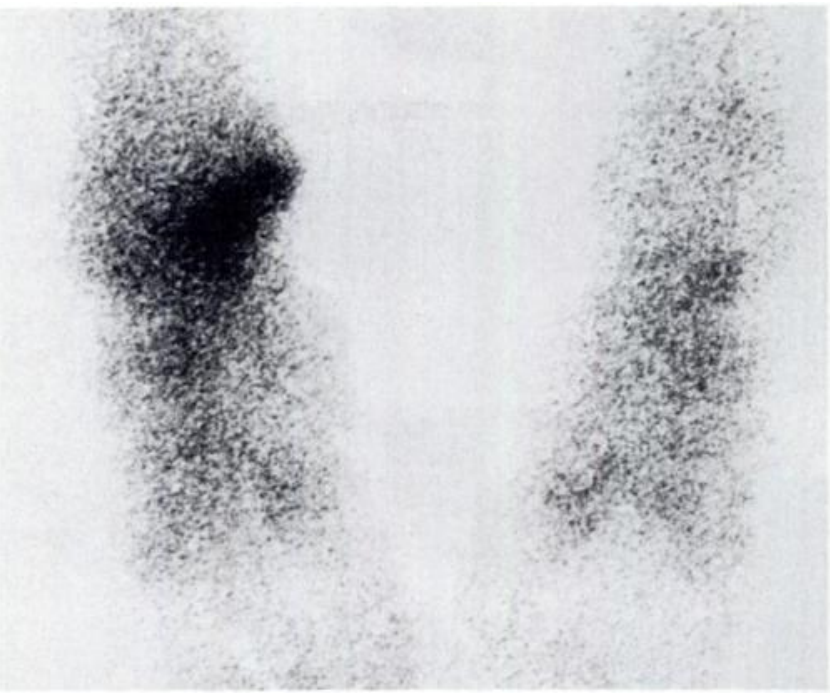

Fig. 5
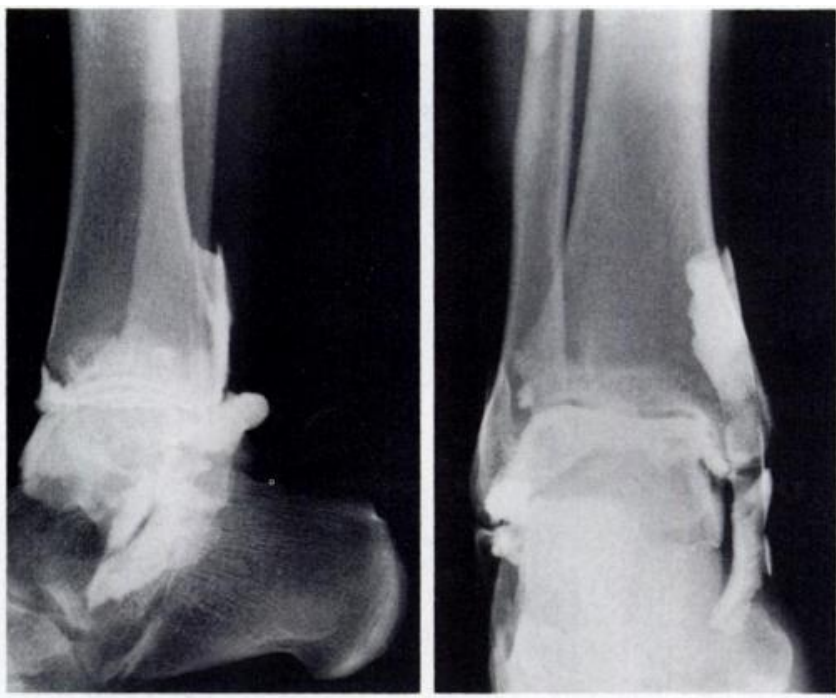

Fig. 6

Case 3. Figure 5 - This 21 -year-old footballer with an eversion ankle injury and persistent pain had a plain radiograph which showed no bony injury; his foot scan, however, showed increased activity localised over the medial capsule and medial collateral ligament. Figure 6 - Ankle arthrograms show contrast leaking into the tibialis posterior tendon sheath, indicating rupture of the medial capsule.

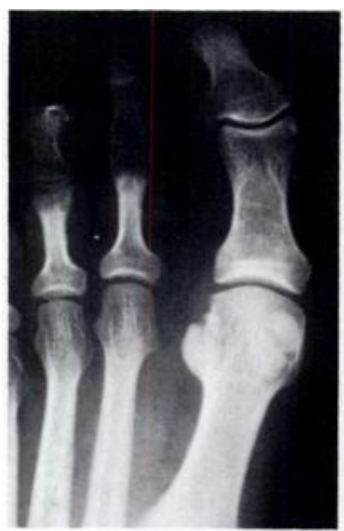

Fig. 7

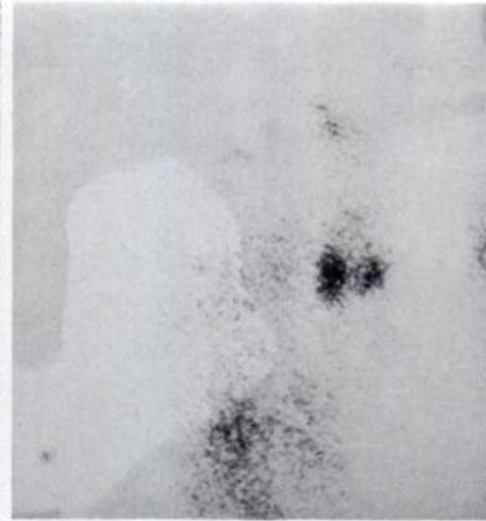

Fig. 8

Case 4. A 45-year-old woman complained of a painful big toe after an injury. A plain radiograph showed both sesamoids divided. A bone scan confirmed fresh fractures with increased activity.

tis secondary to hindfoot fractures; one of these scans showed increased activity in the subtalar joint, indicating delayed union.

\section{Non-traumatic conditions}

Inflammation. Twenty-five patients had scans to exclude bone or joint infection. Six had positive scans; five of these had non-infective arthritis (two with Reiter's disease, one with rheumatoid arthritis and two with osteoarthritis). In one patient the scan confirmed active infection in an area of the lower tibia, which was also lucent in the plain radiographs.

Spontaneous foot pain. Nine patients had no obvious cause for their foot pain and plain radiographs which were normal or equivocal. Seven of these had a normal scan and in all seven the pain settled spontaneously. Both patients with positive scans were subsequently found to have benign tumours - an osteoid osteoma in one and a haemangioma in the other. On reviewing the plain radiographs the osteoid osteoma was visible, though it had at first been missed.

Tarsal coalition. Three young adults each with a painful, stiff flat foot were scanned to exclude a tarsal coalition. One had increased activity in the calcaneonavicular area, suggesting a coalition. On reviewing her radiographs an extra ossicle could be seen between the calcaneus and the navicular; its significance had not been appreciated prior to the scan (Figs 12 and 13). 


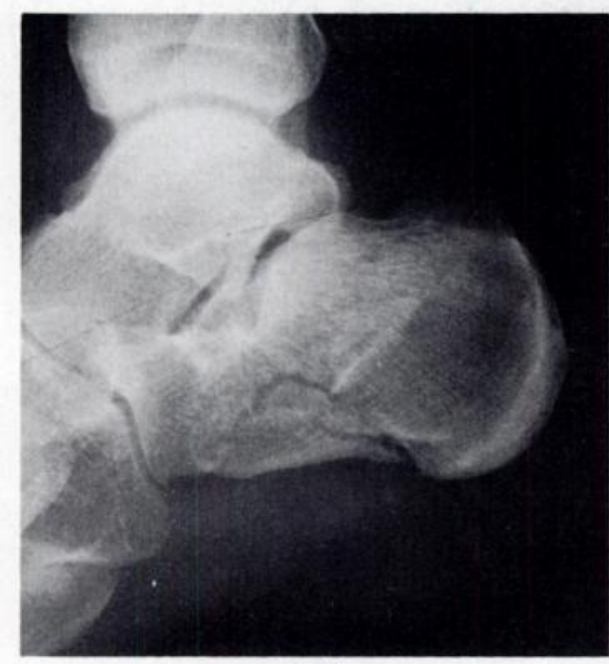

Fig. 9

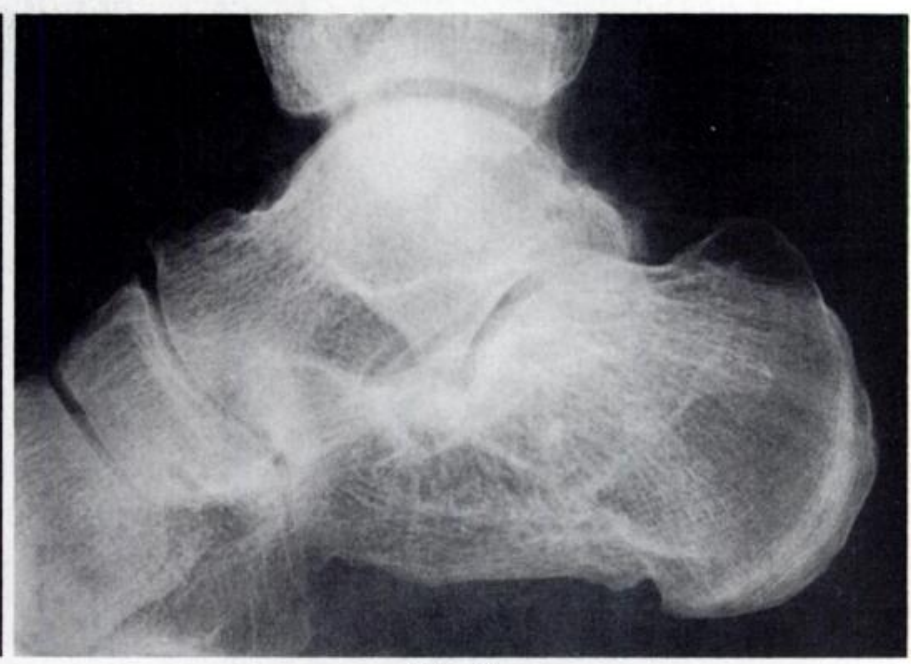

Fig. 10

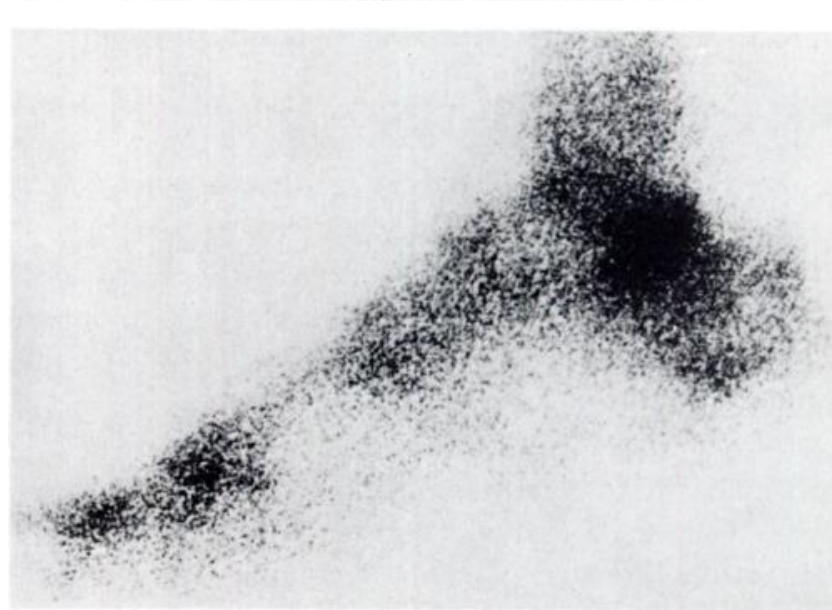

Fig. 11

Case 5. Figure 9 - A 30-year-old man sustained a fractured calcaneus from a fall. Figure 10 - Eighteen months later the fracture was united. Figure $11-$ As he complained of persistent foot pain, a scan was taken which showed increased activity in the subtalar joint, indicating subtalar arthritis.

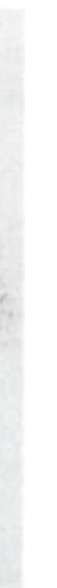




\section{DISCUSSION}

After an injury many patients have a positive bone scan. Interpretation of this scan may be difficult and should be undertaken only in conjunction with the clinical findings and plain radiographs. In the series we have described, none of the patients with a normal scan developed a significant abnormality in their feet; this suggests that scanning may be used as a screening test for bone and soft-tissue pathology in the feet.

A bone scan is a valuable adjunct in the diagnosis of foot disorders which do not show well on plain radiographs. It also often reveals abnormalities before there are any radiographic changes. In the present series scanning was particularly useful in sportsmen with capsular or ligament injuries who had returned to sport after only a brief period of rest or treatment and who then complained of persistent pain. The bone scan showed markedly increased activity localised to the injured area. Clearly soft-tissue trauma, even in the absence of bony injury, can be revealed by increased activity on a bone scan. The reason for this increased activity is not clear. It was present in both the blood pool phase and the delayed images and so represents more than increased vascularity due to acute inflammation. We feel that the use of bone scanning in soft-tissue trauma merits further investigation.

Scanning was useful in the early diagnosis of arthritis of the subtalar joint, where radiographs are often difficult to interpret. In stress fractures the bone scan was also useful because it was positive before radiographic changes were apparent. A fractured sesamoid is, of course, easily seen on a plain radiograph, but it can be confused with a bipartite sesamoid, a very common anomaly, occurring in up to $35 \%$ of people (Helal 1981). A bone scan will show increased activity with a fresh fracture but not with a bipartite sesamoid.

A bone scan also shows increased activity with reflex sympathetic dystrophy, allowing confirmation of the diagnosis. It is important, however, to differentiate between normal disuse activity secondary to immobilisation and a true reflex sympathetic dystrophy.
In the former the increased activity is primarily in the metaphyseal region, while in the latter it is generalised. The scan is particularly useful in the early stages, since it is positive before there are radiographic changes. With active infections also increased activity is seen on the scan before it is on radiographs and a normal scan makes the diagnosis of infection very unlikely. The increased activity with tarsal coalition has been attributed to abnormal stresses passing through the tarsal bones and leading to increased bone turnover; it may therefore occur with fibrous as well as bony coalitions.

Finally, a bone scan may be used as a screening investigation in patients with spontaneous foot pain for which there is no obvious cause. This series has no false negatives, confirming the view that a negative scan excludes significant pathology, whereas a positive investigation highlights and localises lesions which may otherwise have been missed.

\section{REFERENCES}

Galasko CSB, Weber DA, eds. Radionuclide scintigraphy in orthopaedics. Current problems in orthopaedics. Edinburgh, etc: Churchill Livingstone, 1984.

Goldman AB, Pavlov H, Schneider R. Radionuclide bone scanning in subtalar coalitions: differential considerations. AJR 1982;138: 427-32.

Helal B. The great toe sesamoid bones: the lus or lost souls of Ushaia. Clin Orthop 1981;157:82-7.

Hughes S. Radionuclides in orthopaedic surgery. J Bone Joint Surg [Br] 1980;62-B:141-50.

Kozin F, McCarty DJ, Sims J, et al. The reflex sympathetic dystrophy syndrome. I. Clinical and histologic studies: evidence for bilaterality, response to corticosteroids and articular involvement. Am J Med 1976a;60:321-31.

Koxin F, Genant HK, Bekerman C. et al. The reflex sympathetic dystrophy syndrome. II. Roentgenographic and scintigraphic evidence of bilaterality and of periarticular accentuation. Am J Med 1976b;60:332-8.

Sewell JR, Black CM, Chapman AH, Statham J, Hughes GR, Lavender JP. Quantitative scintigraphy in diagnosis and management of plantar fasciitis (calcaneal periostitis): concise communications. $J$ Nucl Med 1980;21:633-6.

Tannenbaum H, Esdaile J, Rosenthall L. Joint imaging in regional migratory osteoporosis. $J$ Rheumatol 1980;7:237-44. 